Mon. Not. R. Astron. Soc. 000, 000-000 (0000) Printed 22 November $2018 \quad$ (MN LATEX style file v2.2)

\title{
The star formation efficiency and its relation to variations in the initial mass function
}

\author{
Paul C. Clark ${ }^{1 \star}$, Ian A. Bonnell ${ }^{2} \&$ Ralf S. Klessen ${ }^{1}$ \\ 1 Institut für Theoretische Astrophysik, Universität Heidelberg, Albert-Ueberle-Straße. 2, Heidelberg, Germany \\ 2 School of Physics \& Astronomy, University of St Andrews, North Haugh, St Andrews, Fife, KY16 9SS, UK
}

22 November 2018

\begin{abstract}
We investigate how the dynamical state of a turbulently supported, $1000 \mathrm{M}_{\odot}$, molecular cloud affects the properties of the cluster it forms, focusing our discussion on the star formation efficiency (SFE) and the initial mass function (IMF). A variety of initial energy states are examined in this paper, ranging from clouds with $\left|E_{\text {grav }}\right|=$ $0.1 E_{\text {kin }}$ to clouds with $\left|E_{\text {grav }}\right|=10 E_{\text {kin }}$, and for both isothermal and piece-wise polytropic equations of state (similar to that suggested by Larson). It is found that arbitrary star formation efficiencies are possible, with strongly unbound clouds yielding very low star formation efficiencies. We suggest that the low star formation efficiency in the Maddelena cloud may be a consequence of the relatively unbound state of its internal structure. It is also found that competitive accretion results in the observed IMF when the clouds have initial energy states of $\left|E_{\text {grav }}\right| \geqslant E_{\text {kin }}$. We show that under such conditions the shape of the IMF is independent of time in the calculations. This demonstrates that the global accretion process can be terminated at any stage in the cluster's evolution, while still yielding a distribution of stellar masses that is consistent with the observed IMF. As the clouds become progressively more unbound, competitive accretion is less important and the protostellar mass function flattens. These results predict that molecular clouds should be permeated with a distributed population of stars that follow a flatter than Salpeter IMF.
\end{abstract}

\section{INTRODUCTION}

The internal motions in giant molecular clouds (GMCs) are 'dominated by supersonic turbulence (for example, Larson 1981; Mvers 1983; Dame et al. 1986; Solomon et al. 1987; Hever \& Brunt 2004). These motions leave their imprint on the cloud's internal structure (Mac Low \& Klessen 2004; Elmegreen \& Scalo 2004), resulting in dense regions, often referred to as clumps or cores, that are observed to have a wide range of dynamical states Bally et al. 1987; Williams et al. 1994). The aim of this paper is to explore how the initial dynamical state of star forming regions can influence both their final stellar mass functions and their star formation efficiency (SFE).

Clark \& Bonnell (2004) suggested that stars can form in dynamically unbound molecular regions, where the internal turbulent motions are in excess of the region's gravitational energy. Depending on the scale, such regions can be star forming cores or clouds, and could thus give rise to a wide range of cluster sizes. For dynamically unbound regions to be capable of producing stars, they need to contain multiple thermal Jeans masses, or undergo better than isothermal cooling during the shock compression phase (e.g. Chapman et al. 1992; Bhattal et al. 1998; Larson 2005). This is simply because isothermal shock compression (in 1-D) does not significantly increase the number of Jeans masses in the shock layer (Elmegreen \& Elmegreen 1978; Doroshkevich 1980; Lubow \& Pringle 1993; Clarke 1999).

This fact is apparent in the numerical studies of turbulent molecular clouds, which show that the number of stars is always comparable to the number of initial Jeans masses in the simulation, despite the huge density contrast produced by the turbulence or colliding flows (Klessen et al. 2000; Gittins et al. 2003; Clark \& Bonnell 2005; Larson 2007).

Dynamically unbound star forming regions have three important features. First, star formation occurs on a similar timescale to the dispersion of the region. Such a picture is consistent with the rapid star formation model (Vazquez-Semadeni, Passot \& Pouquet 1995; Ballesteros-Paredes, Hartmann \& Vázquez-Semadeni 1999; Elmegreen 2000; Hartmann, Ballesteros-Paredes \& Bergin 2001; Pringle, Allen \& Lubow 2001). This process has gained recent support from the studies of Glover \& Mac Low (2007a,b), which show that $\mathrm{H}_{2}$ formation can occur on a dynamical time in both turbulent or freely collapsing gas. The second feature is that unbound clouds can naturally result in lower star formation efficiencies than their bound counterparts, simply because much of the gas is unable to rid itself of the excess kinetic energy and achieve a bound state. The efficiencies can range from 50 percent at protostellar core scales Clark \& Bonnell 2004), to between 5 and 10 percent at the larger scales 

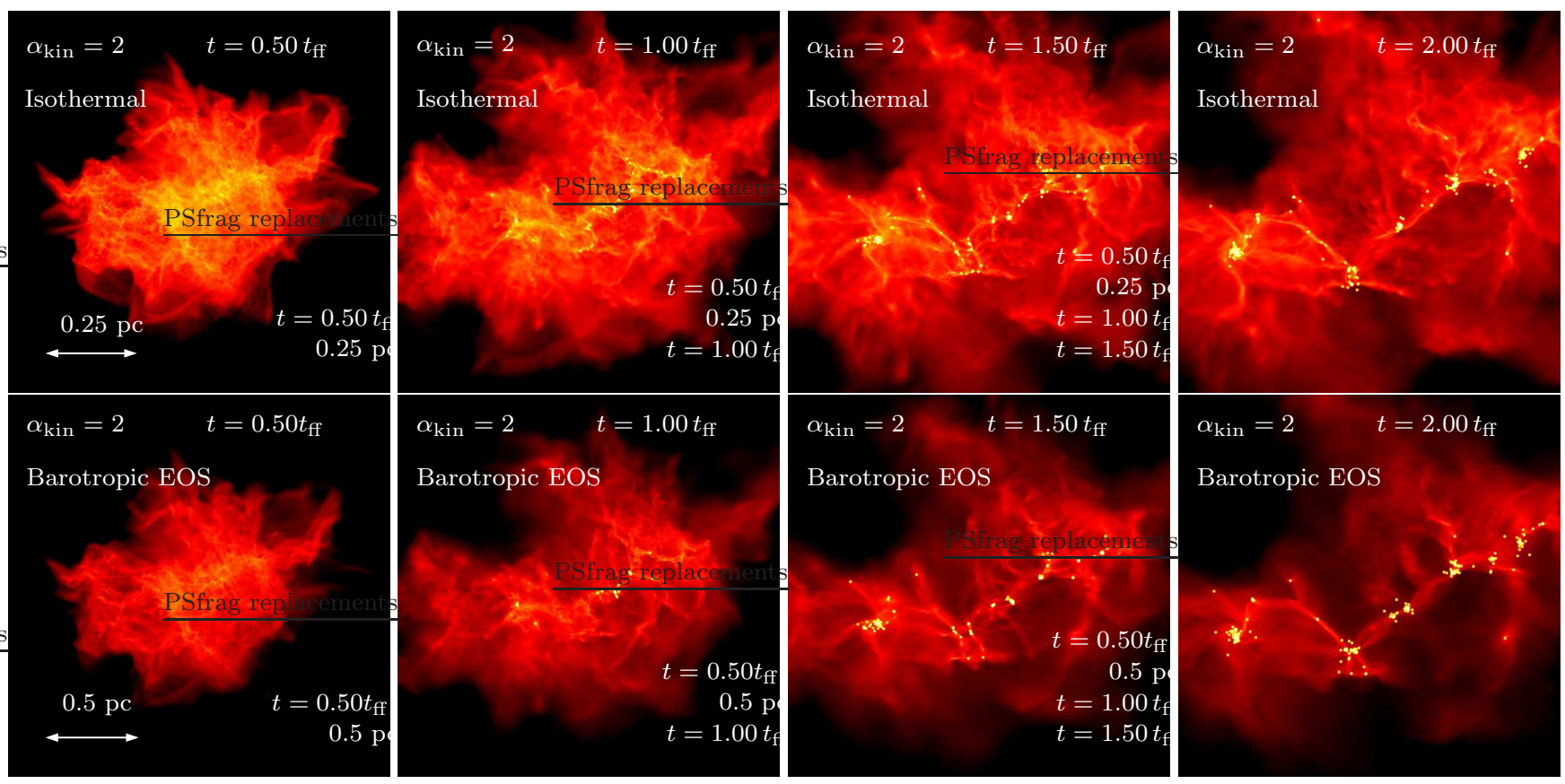

Figure 1. Evolution of the column density distribution in the gas for 2 of the $\alpha_{\text {kin }}=E_{\text {kin }} /\left|E_{\text {grav }}\right|=2$ simulations, for an isothermal (top) and a piece-wise barotropic EOS (bottom), similar to that discussed by Larson (2005). Both the simulations have the same initial turbulent field. The colour table in the images is stretched (logarithmically) over 5 orders of magnitude in column density, ranging from 0.001 to $100 \mathrm{~g} \mathrm{~cm}^{-2}$

of a GMC (Clark et al. 2005). Finally, Clark et al. (2005) also found that at the GMC scale, the unbound cloud can produce a series of clusters that reproduce some of the features found in OB sub-groups.

The aim of this present study is two-fold. First, we wish to explore how the initial energy balance in the cloud can affect the star formation efficiency, extending the work of Clark \& Bonnell (2004) and Clark et al. (2005) (see also Klessen, Heitsch \& Mac Low 2000, Heitsch. Mac Low \& Klessen 2001, Vázquez-Semadeni. Ballesteros-Paredes \& Klessen 2003). Secondly, we wish to look at how the mass function of the protostars is influenced by such an environment. When competitive accretion (Zinnecker 1982; Bonnell et al. 2001a; Bonnell et al. 2001b) is the dominant process controlling the distribution of stellar masses, it has been shown that the break in the protostellar mass function, and thus the characteristic mass, depends on the mean Jeans mass (Klessen et al. 1998; Klessen \& Burkert 2000, 2001; Klessen 2001; Jappsen et al. 2005; Bonnell, Clarke \& Bate 2006). For clouds which are out of global dynamic stability, the mean density (and thus the Jeans mass) are constantly changing. As such, the ability of competitive accretion to produce an IMF-like protostellar mass function under these conditions is not clear.

To address these questions we perform a series of numerical simulations which follow the evolution of $1000 \mathrm{M}_{\odot}$ clouds, in which the initial ratio of turbulent kinetic energy to gravitational potential energy is systematically varied. These clouds, which are similar to those studied by Bonnell. Bate \& Vine (2003), can be thought of as overdensities in a larger GMC, which still contain residual kinetic energy from the supersonic flows that created them. Further details of the calculations are given in Section 2 , and we discuss the star formation efficiency and mass functions from these clouds in sections 3 and 4 respectively. The implications of our results for star formation in GMCs in general are presented in Section 5 and a brief summary of our findings is given in Section 6

\section{THE SIMULATIONS}

\subsection{Computational Method and Cloud set-up}

The evolution of the gas was modelled using the Smoothed Particle Hydrodynamics (SPH) method (for a description of this technique, see for example Benz 1990, Monaghan 1992, Monaghan 2005), and the simulations we present here were performed on the UK Astrophysical Fluids Facility (UKAFF), a collection of IBM Power 5 nodes. The code utilises adaptive smoothing lengths for the gas particles, allowing high density regions to be followed with increased resolution. Gravity is calculated using a binary tree algorithm, which also serves to construct SPH neighbour lists.

The clouds in this study have a mass of $1000 \mathrm{M}_{\odot}$, which we model using $2 \times 10^{6} \mathrm{SPH}$ particles in an initially uniform density sphere (see below for the initial density values). With this set-up, the simulations have a mass resolution of $0.05 \mathrm{M}_{\odot}$, sufficient to capture the fragmentation of the gas to below the hydrogen burning limit Bate \& Burkert 1997). The clouds are also given a turbulent velocity field, which is allowed to decay freely via shocks. The turbulent velocity fields imposed here are described by a power spectrum of $P(k) \propto k^{-4}$, which is consistent with the turbulent motions observed in molecular clouds (Larson 1981; Mvers \& Gammie 1999; Hever \& Brunt 2004). In this pa- 
per, we investigate the systematic changes that occur in the properties of the star formation as the dynamical state of a cloud is altered. We therefore use the same realisation of the turbulent field for most of the simulations. We do however perform several simulations with a different turbulent field, to check that any systematic trends in the star formation properties are not due to any specific feature of the initial turbulent field. To adjust the dynamical state of the clouds, we change the initial ratio of kinetic to gravitational potential energy, $\alpha_{\text {kin }}=E_{\text {kin }} /\left|E_{\text {grav }}\right|$, to vary from 10 to 0.1 .

We adopt two types of equation of state (EOS) in this study: the first assumes the gas is isothermal and the second is a barotropic EOS, similar to that proposed by Larson (2005). In the isothermal gas, we set up the gas to have a Jeans mass of roughly $1 \mathrm{M}_{\odot}$, such that the cloud has initially 1000 Jeans masses. In these isothermal runs, the clouds have a temperature of $10 \mathrm{~K}$, and start at a density of $1.45 \times 10^{19} \mathrm{~g} \mathrm{~cm}^{-3}$. For the barotropic EOS, we use

$T=T_{\mathrm{c}}{\frac{\rho}{\rho_{\mathrm{c}}}}^{-0.25}$, for $\rho<\rho_{\mathrm{c}}$

and then,

$T=T_{\mathrm{c}}$, for $\rho>\rho_{\mathrm{c}}$,

where $\rho_{\mathrm{c}}=5.5 \times 10^{-19} \mathrm{~g} \mathrm{~cm}^{-3}$ and $T_{\mathrm{c}}=7.5 \mathrm{~K}$. The values for $T_{\mathrm{c}}$ and $\rho_{\mathrm{c}}$, ensure that at the point where the barotropic EOS enters the isothermal regime (that is, at densities higher than $\rho_{\mathrm{c}}$ ), the means Jeans mass is roughly the same as the initial value (roughly $1 \mathrm{M}_{\odot}$ ) found in the pure isothermal clouds. The barotropic EOS clouds start at a density of $1.83 \times 10^{-20} \mathrm{~g} \mathrm{~cm}^{-3}$ and a temperature of $17.6 \mathrm{~K}$.

To model the star formation in this study we use 'sink' particles, as described by Bate et al. (1995), which involves replacing dense, self-gravitating, regions of gas with a point mass. Sink particles are capable of accreting further material and they can interact with the other particles in the simulation via gravity. The sinks are formed in these simulations once a particle and it's neighbours are bound, collapsing, and within an accretion radius, $h_{a c c}$, which is taken here to be $412 \mathrm{AU}$. The sinks are then able to accrete any bound $\mathrm{SPH}$ particles which fall inside the accretion radius. The gravitational interactions between the sink particles and all other particles in the simulation are also smoothed to $h_{a c c}$. Due to the size of the sink particles in this study, it should be stressed that their mass functions presented in this paper should not be thought of as a stellar object IMF. Instead, we suggest that they are more representative of a 'system' mass function (Kroupa, Gilmore \& Tout 1991; Kroupa 2002). As such, we will refer to them simply as 'sink' mass functions in the remainder of this paper.

\subsection{General Evolutionary Properties}

For the clouds with $\alpha_{\text {kin }} \geqslant 1$, we run the simulations for two initial free-fall times. In our discussion of these simulations, we will use the properties at the end of the run. For our weakly supported clouds, which have $\alpha_{\text {kin }}=0.1$, we only follow the evolution for 1 initial free-fall time. In these latter simulations, the extreme dynamics and environment make them computationally very expensive. However, since they also evolve faster, one gains enough information for our present study.

In Figure 1 we show column density snapshots from two of the simulations in this study. Both have the same turbulent velocity seed, and have $\alpha_{\text {kin }}=2$. The evolution of the isothermal cloud is shown in the upper panels, and the evolution of a cloud with a barotropic EOS is shown in the bottom panels. The expansion of the cloud is apparent in the images, and one can see that the clusters move apart as they form, in contrast to the merging process that has been documented in studies where $\alpha_{\text {kin }}=1$ initially (Bonnell et al. 2003; Bonnell, Vine \& Bate 2004). One can also see that the two different EOSs look very similar, since the sites of star formation are selected by the turbulent velocity field.

\section{THE STAR FORMATION EFFICIENCY}

We will first look at how the star formation efficiency (SFE) in a molecular region can be controlled by its dynamical state. We define the SFE in this study as,

$\mathrm{SFE}=\frac{M_{\text {sinks }}}{\left(M_{\mathrm{gas}}+M_{\mathrm{sinks}}\right)}$,

where $M_{\text {sinks }}$ and $M_{\text {gas }}$ are the total mass in sink particles and gas, respectively. In Figure 2 we plot the SFE as function of time for all the simulations with $\alpha_{\text {kin }}=$ $E_{\text {kin }} /\left|E_{\text {grav }}\right| \geqslant 1$. The isothermal calculations are on the left and the barotropic EOS are on the right. We plot time in units of both the initial free-fall time of the clouds, and in years. The free-fall time is $1.75 \times 10^{5} \mathrm{yr}$ in the isothermal runs, and $4.92 \times 10^{5} \mathrm{yr}$ in the barotropic EOS.

The plots show that a wide range of SFEs are possible, depending on the initial dynamical state of the cloud, with the SFE decreasing with increasing $\alpha_{\text {kin }}$. These calculations also suggest that star formation is able to take place under a variety of conditions, provided that the region has a sufficient number of Jeans masses at the outset. While the SFEs in our simulations are clearly not converged, and without feedback one would not expect them to be, they do demonstrate a clear trend with the initial level of turbulent support. One must also consider here that the SFEs given in Figure 2 are upper limits to the true value, since sink particles do not contain any model of the feedback that may be important in inhibiting further accretion. Also, not all the gas accreted by the sinks would be expected to find its way into stars, especially for the high-mass objects (see Zinnecker \& Yorke 2007 for further discussions).

The barotropic and isothermal EOSs give very similar results, in terms of the SFE evolution as a function of the initial free-fall time. This suggests that the regions for collapse are selected for fragmentation at similar times in the evolution, and that these regions proceed directly to collapse on the corresponding free-fall time. The similarity of both the cloud structure and cluster distribution between the two different EOS (Figure 1) also suggests that the general morphology of the region is controlled primarily by the turbulent motions flows, via shock compression and dissipation of kinetic energy. It is also interesting to note just how quickly the SFE drops as the value of $\alpha_{\text {kin }}$ increases. Given that most the structure in a GMC is typically thought to be unbound (Williams et al. 1994), one would expect the 

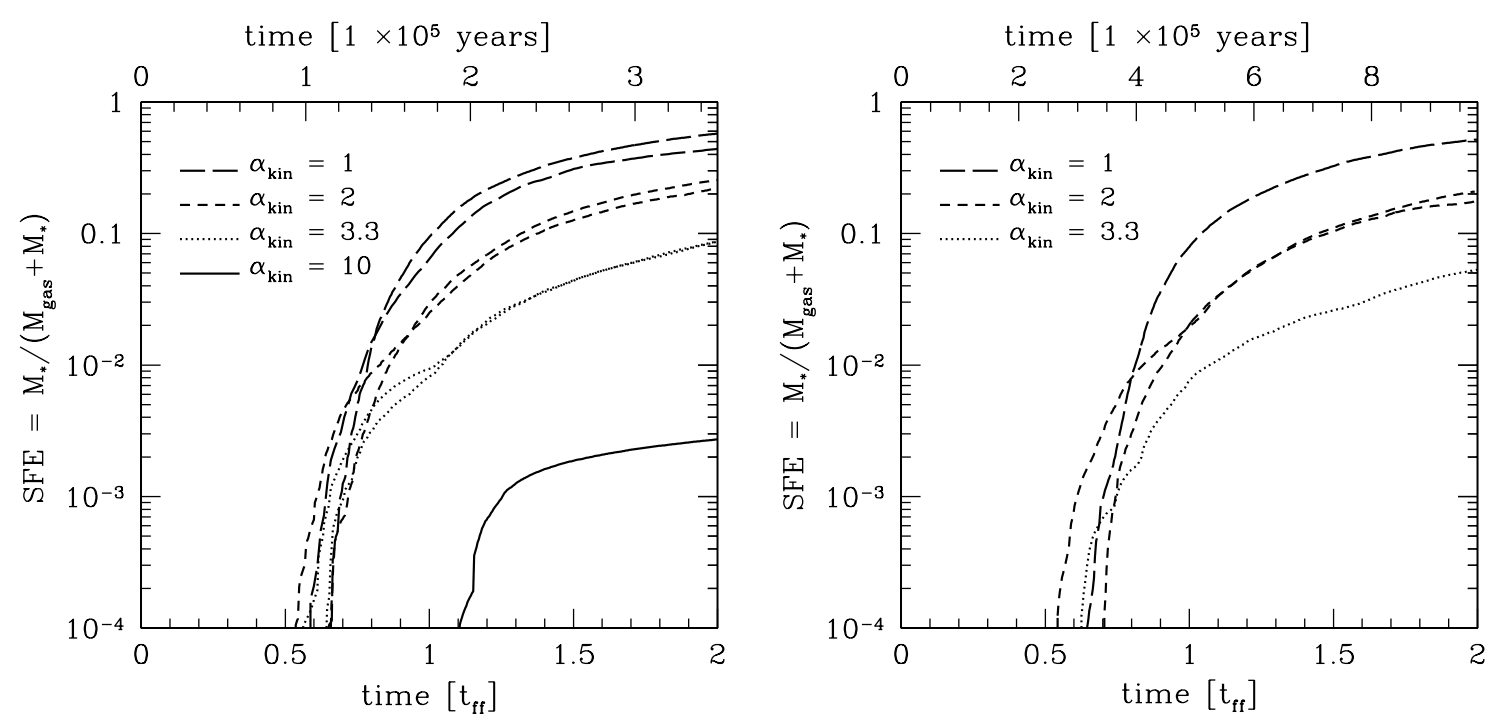

Figure 2. The star formation efficiency, $\mathrm{SFE}=M_{\mathrm{sinks}} /\left(M_{\mathrm{gas}}+M_{\mathrm{sinks}}\right)$, as a function of time for the simulations with $\alpha_{\mathrm{kin}}=$ $E_{\text {kin }} /\left|E_{\text {grav }}\right| \geqslant 1$. Two different random realisations of the initial turbulent velocity field were performed for $\alpha_{\text {kin }}=1,2, \& 3.3$ in the isothermal case, and for $\alpha_{\text {kin }}=2$ in the barotropic case. Time is given in both units of a free-fall in the bottom x-axis and in years in the top x-axis.

majority of star formation in GMCs to only occur in a few regions, in which the local SFE can be quite high.

The weakly supported clouds, naturally, have a much higher rate of star formation. We plot in Figure 3 the $\mathrm{SFE}$ as function of time (free-fall units) for the clouds with $\alpha_{\text {kin }}=0.1$, and for comparison, we also show the SFE curve from the isothermal $\alpha_{\text {kin }}=1$ calculation. Clearly, these weakly supported clouds evolve much faster than their more strongly supported counterparts, reaching 3-4 times the efficiency in only half the time.

\section{THE SINK PARTICLE MASS FUNCTIONS}

As mentioned in Section 1 above, the effect of the competitive accretion process on the IMF in dynamically unbound simulations is still unclear, and needs to be examined. In Figure 4 we plot the sink particle mass function for all the simulations that have $\alpha_{\text {kin }}=E_{\text {kin }} /\left|E_{\text {grav }}\right| \geqslant 1$. The isothermal clouds are shown on the left and the barotropic EOS clouds on the right. Some properties of the sink particles at the end of simulations are given in Table 4 .

The panels on the left-hand side show the sink mass functions for the calculations with $\alpha_{\text {kin }}=1$. These have a similar initial set-up to those performed by Bonnell et al. 2003, 2004, 2006), where it was demonstrated that for a mean initial Jeans of roughly $1 \mathrm{M}_{\odot}$, the resulting sink mass function is similar to the field-star IMF (for the driven turbulent case, see also Klessen 2001). Our simulations yield a similar result. Although the mean initial Jeans mass is much higher in the barotropic EOS simulation, the final result is very similar to that from the isothermal calculation. As has been discussed by others Larson 2005; Jappsen et al. 2005; Bonnell et al. 2006), this is simply because the Jeans mass at the critical density, $\rho_{\mathrm{c}}$, where fragmentation can occur, is similar to that in the isothermal case.
One can see from Figure 4 , that there is a distinct trend in the sink mass functions as the value of $\alpha_{\text {kin }}$ is increased: the mass functions become flatter as the clouds become progressively more unbound. A similar behaviour is seen in calculations of driven turbulence with decreasing driving scale Klessen 2001). This feature is also seen in the barotropic EOS calculations, suggesting that the trend in the mass function is not simply due to the changing Jeans mass in the unbound clouds. One can see this trend in the properties of the sinks, given in table 4 . The quantity $f_{\mathrm{M}}>1 \mathrm{M}_{\odot}$ denotes the fraction of the total mass in sink particles that is made up of sinks with masses $1 \mathrm{M}_{\odot}$ or greater. Mever et al. (2000) performed a similar analysis on a number of local embedded clusters (using the number of stars, rather than the mass). One can see here that the fraction of the mass function comprising sinks of $1 \mathrm{M}_{\odot}$ or more is clearly rising as $\alpha_{\text {kin }}$ is increased, indicating an overall flattening of the mass functon.

There are two effects which will play an important role in shaping the sink mass function in the unbound clouds: the timescale of interactions, and the degree of fragmentation. First, we consider the timescale on which newly formed objects interact with their siblings. In the $\alpha_{\text {kin }}=1$ simulations, the clouds are characterised by a particular density. For the isothermal clouds, this is the initial mean cloud density, while in the barotropic EOS clouds, this is density, $\rho_{\mathrm{c}}$, at which the cooling stops. When fragmentation occurs at this density, the timescale for neighbouring fragments to interact is the same as the timescale for each fragment to collapse to form a sink particle. Naturally, this is just the free-fall time associated with the density where the fragmentation sets in. In contrast, the background density in the unbound simulations is constantly decreasing. The regions which become bound, and so manage to form sink particles, decouple from the surrounding material, which is evolving on progressively longer timescales. As a result, the star-forming regions which 
Table 1. We give here the properties of the sink particles after 2 initial free fall times have elapsed, for all the simulations with $\alpha_{\text {kin }} \geqslant 1$. For comparison, we also show the results from one of the simulations with $\alpha_{\text {kin }}=0.1$, with the results taken after 1 initial free-fall time (see Section 2.2 for details). The equation of state (EOS) is shown in column 2, with 'iso' denoting isothermal, and 'lar' denoting a piece-wise barotropic EOS (see Section 2 and Equations 1 and 21 that is similar to that suggested by Larson (2005). The seed column shows which of the two random realisations of the initial turbulent velocity field was used for that calculation. $N_{*}, M_{*, \text { total }}$ and $\bar{M}_{*}$ are the total number and mass in sink particles and mean sink particle mass, respectively, with $\bar{M}_{*}=M_{*, \text { total }} / N_{*}$. Finally, $f_{\mathrm{M}>1 \mathrm{M}_{\odot}}$ denotes the fraction of the total mass in sinks which is made up of sinks with masses of $1 \mathrm{M}_{\odot}$ or greater.

\begin{tabular}{rccrrcc}
\hline$\alpha_{\text {kin }}$ & EOS & Seed & $N_{*}$ & $M_{*, \text { total }}$ & $\bar{M}_{*}$ & $f_{\mathrm{M}>1 \mathrm{M}_{\odot}}$ \\
\hline \hline 1.0 & iso & 1 & 1236 & 574.85 & 0.47 & 0.54 \\
2.0 & iso & 1 & 555 & 255.50 & 0.46 & 0.69 \\
3.3 & iso & 1 & 120 & 87.54 & 0.73 & 0.70 \\
10.0 & iso & 1 & 2 & 2.72 & 1.36 & 1.00 \\
1.0 & lar & 1 & 1029 & 518.72 & 0.50 & 0.57 \\
2.0 & lar & 1 & 442 & 208.71 & 0.47 & 0.75 \\
3.3 & lar & 1 & 57 & 53.01 & 0.93 & 0.84 \\
1.0 & iso & 2 & 899 & 574.85 & 0.47 & 0.54 \\
2.0 & iso & 2 & 440 & 255.50 & 0.46 & 0.69 \\
3.3 & iso & 2 & 157 & 87.54 & 0.73 & 0.70 \\
2.0 & lar & 2 & 314 & 119.06 & 0.56 & 0.68 \\
0.1 & iso & 1 & 1073 & 339.00 & 0.32 & 0.28 \\
\hline
\end{tabular}

form in the unbound clouds are able to use up their gas reservoir without as much influence from their neighbours. The unbound clouds hence move towards a more isolated mode of star formation, whereby individual regions of star formation evolve independently.

The second effect which shapes the mass function is the degree of fragmentation. From Figure 4 , one can see that the more unbound simulations still form fairly high-mass sinks. What makes the mass function progressively flatter is the lack of lower-mass objects, since the clouds with higher levels of turbulent energy form systematically fewer bound objects. This occurs because many regions take several sound crossing times to become bound, during which time the internal energy has erased much of the structure. Naturally, this is more pronounced in the barotropic EOS calculations, since the gas heats up as it becomes less dense. The lack of fragmentation within the star forming regions reduces the role of competitive accretion.

A similar effect is seen in the driven turbulent simulations of Klessen (2001). When the turbulent driving scale is small, the stars tend to form in relative isolation. Since they have little gravitational interaction with their siblings, the resulting mass functions are flat. In contrast, large scale driving leads to a highly clustered environment, in which competitive accretion is important. Consequently, these latter simulations produce mass functions which are more similar to the IMF.

After considering the unbound clouds, one may expect that the clouds with $\alpha_{\text {kin }}=10$ to result in mass functions which are very steep. Figure 5] shows that this is not the case. In these clouds, where the collapse proceeds in a quasihomologous fashion, the sinks interact strongly with their

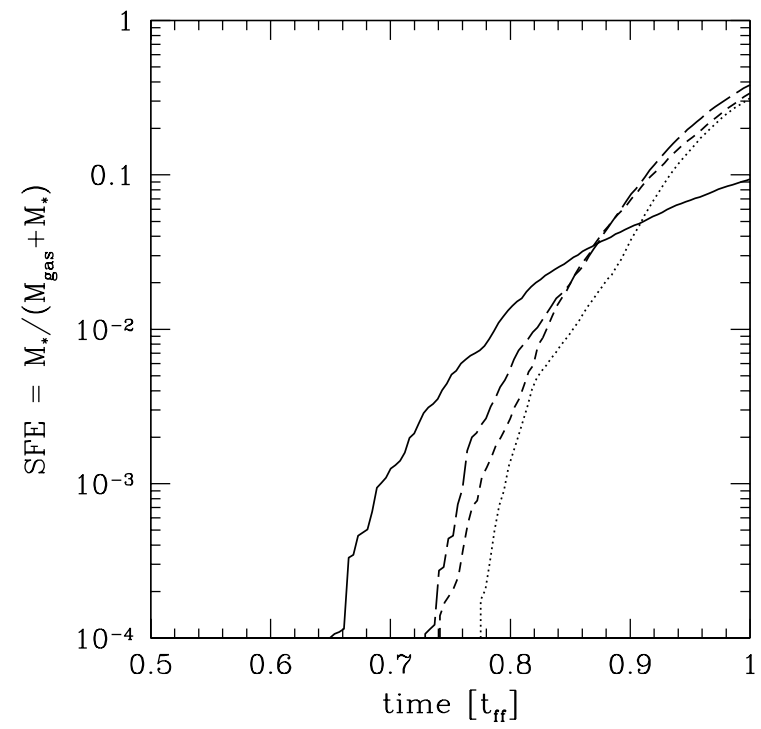

Figure 3. The mass in sinks as a function of time for the clouds with $\left.\alpha_{\text {kin }}=E_{\text {kin }} /\left|E_{\text {grav }}\right|=0.1\right)$. The long-dashed and shortdashed lines show the $S F E(t)$ for two isothermal runs which have different initial random realisations of the turbulent velocity field. The dotted line traces the $\operatorname{SFE}(t)$ for the simulation with the barotropic EOS. For comparison, we plot the $\operatorname{SFE}(t)$ from the isothermal $\alpha_{\text {kin }}=1$ simulation (solid line), which shares the same seed as the $\alpha_{\text {kin }}=0.1$ denoted by the long-dashed line.

neighbours, which leads to a mass spectrum that is broadly similar to the IMF. Indeed, it has been shown (Zinnecker 1982; Bonnell et al. 2001b) that the mass function should never get infinitely steep under these conditions, with an asymptotic limit for the slope reaching -2.5. Again we see that the isothermal and barotropic EOSs give very similar results.

Lastly, we note that when competitive accretion occurs in clouds with $\alpha_{\text {kin }} \leqslant 1$, the mass function of the accreting objects always has roughly the same shape. This in demonstrated in Figure 6] which shows the evolution of the mass function for one of the isothermal runs with $\alpha_{\text {kin }}=1$. This is an important feature of the mass function in these cloud, since it means that the star formation need not be terminated at some special time, but can indeed be terminated at any point in the evolution and will still give the correct IMF.

One can see why the shape of the mass function remains constant in the $\alpha_{\text {kin }} \leqslant 1$ calculations by considering the time-scales for accretion and fragmentation in these calculations. As discussed by Bonnell et al. (2001a), the accretion time-scale in such clouds is comparable to the free-fall time. Further, the crossing time, which dictates the decay timescale of the turbulence in the gas (Mac Low et al. 1998), is related to the free-fall time in these clouds by,

$\frac{t_{\mathrm{ff}}}{t_{\mathrm{cr}}}=\left(\frac{3 \pi}{32 G \rho}\right)^{1 / 2} \frac{2 r}{v_{\mathrm{rms}}}=\frac{\pi}{\sqrt{10}} \alpha_{\mathrm{kin}}^{1 / 2}$,

where $r$ is the radius of the cloud, $v_{\mathrm{rms}}$ is the root-meansquare of the turbulent velocities, and $\rho$ is the mean density in the cloud. Naturally, this is also the average time-scale on which regions of gas will lose their turbulent support and 
start to collapse, and as such can be thought of as the fragmentation time. Since the time-scales for the accretion and fragmentation are similar throughout the cloud's evolution, the rate at which new sinks form is then similar to rate at which mass is added to the pre-existing sinks via accretion. Thus, both the Salpeter section of the mass function and the flatter section at lower masses grow together, which maintains the shape of the mass distribution.

In the contrasting picture to competitive accretion, in which the stellar masses are set purely by the fragmentation of the molecular cloud, it is not clear that the emerging IMF would be time independent. Clark, Klessen \& Bonnell (2007) pointed out that any stellar mass function that results from the collapse of a bound distribution of cores will change with time, provided that the time-scale for the cores to collapse is a function of their mass. While this may not be a problem for the Salpeter (1955) section of the core mass function, the time-scale issue may change the mapping between the observed cores and final stars at lower masses (for example, see André et al. 2007).

\section{DISCUSSION}

The results of this paper show that arbitrarily low star formation efficiencies are possible with just slight variations in the initial kinematic state of the cloud or star forming region. As determinations of the total cloud masses and kinetic energy content are uncertain, such variations are easily within our current understanding of molecular clouds. For example, an initial $\alpha_{\text {kin }}=0.3$ corresponds to a mass three times less than what is required to be bound or equivalently an internal velocity dispersion 1.8 times higher.

In order to fully understand the significance of these results, we need to put them in a broader context of cloud formation and evolution. Recent work has shown that a dynamical formation mechanism for molecular clouds is likely due to some larger scale compression Ballesteros-Paredes et al. 1999; Hartmann et al. 2001; Bonnell et al. 2006; Dobbs et al. 2006; Vázquez-Semadeni et al. 2006). Shocks induced by spiral arms, or supernovae are two such possibilities. In these cases, there is no reason to assume that the clouds are gravitationally bound on the largest scales. Instead, as local regions become close to being gravitationally bound due to the compression Klessen et al. 2005; Ballesteros-Paredes 2006), then star formation is initiated on this local scale (Bonnell et al 2006). The star formation is likely to be inefficient except in those regions which are strongly gravitationally dominated, resulting in a low overall star formation efficiency.

Although the above scenario is dynamical in nature, it can easily be mistaken as being slow in terms of the star formation rate per free-fall time (Krumholz \& Tan 2007). The primary reason for this is that an observer is unlikely to see only the final stages of the process but is equally likely to observe any intermediate state in the evolution. Thus what we need to compute is the time-averaged star formation efficiency for a given cloud. For example, given the end results presented in Figure 2 the time averaged star formation efficiency per free-fall time (as defined from the initial density) for the cases of $\alpha_{\text {kin }}=1,0.5,0.3$ are
$\mathrm{SFE} / t_{\mathrm{ff}} \approx 0.18,0.07$, and 0.02 , respectively. These are significantly lower than just the final star formation efficiency and could be taken to imply that star formation in these simulations was slow. Furthermore, we need also consider the formation timescale of the cloud which effectively doubles the cloud lifetime (Ballesteros-Paredes et al. 1999) and thus halves the above estimates of the star formation efficiency per free-fall time to $0.09,0.035$ and 0.01 .

The results of this paper may also provide an explanation for the relative lack of star formation in the Madellena GMC. We show here that star formation becomes less efficient and more distributed as the clouds become more unbound. Similarly, Williams et al. (1994) have demonstrated that the structure in the Maddelena cloud is significantly unbound, and such structures have estimated masses in the range from around 2 to $1000 \mathrm{M}_{\odot}$. We hence suggest that the highly unbound and dynamic environment in the Maddelena cloud may be responsible for the very low level of star formation.

We also predict that dynamically unbound regions should produce more isolated stars that follow a flatter IMF than those in the bound clustered environments. Given the unbound state of the majority of the structure in, for example, the Orion A cloud (Bally et al. 1987) or the Rosette molecular cloud (Williams et al. 1994), we would predict that a low level of star formation should permeate most molecular clouds, providing that the dense regions have a sufficient number of Jeans masses. Current observations suggest that this is indeed the case. The 2MASS and Spitzer surveys have revealed distributed populations of stars in a number of nearby star-forming regions (Carpenter 2000; Allen et al. 2007), and the data suggest a smooth transition between 'distributed' and 'clustered' star formation Allen et al. 2007). From the wide variety in dynamical states for the sub-structure in molecular clouds, our results would also suggest a smooth transition between heavily clustered environments and regions of relatively isolated star formation.

One potential problem with the model we present here is that the resulting field-star IMF in the Galactic disc would become contaminated by the flatter IMFs from the unbound regions. If most star formation occurs in the more massive bound clusters, then competitive accretion is able to produce the observed IMF. In contrast, if the majority of stars instead form in loose associations, in which gravity only dominates at the scale of individual stars or systems, then our results would suggest an integrated IMF that is shallower than the observed distribution. However, the star formation efficiency is also important. Although the more unbound clouds produce flatter IMFs, they do so with a lower efficiency, and thus the overall effect of the distributed population is not clear. Estimates for the distributed population in several nearby star forming regions suggest that these objects comprise around 20 to 30 percent of the host cloud's star formation (Allen et al. 2007), which will also include any stars that have left dense clusters or groups. This implies that the contamination problem is not too severe. We also note that Lada \& Lada (2003) estimate that the majority of stars do form in clusters.

Lastly, we issue a word of caution when interpreting the mass functions presented in this study. Sink particles only store the potential mass reservoir for the protostar, or pro- 


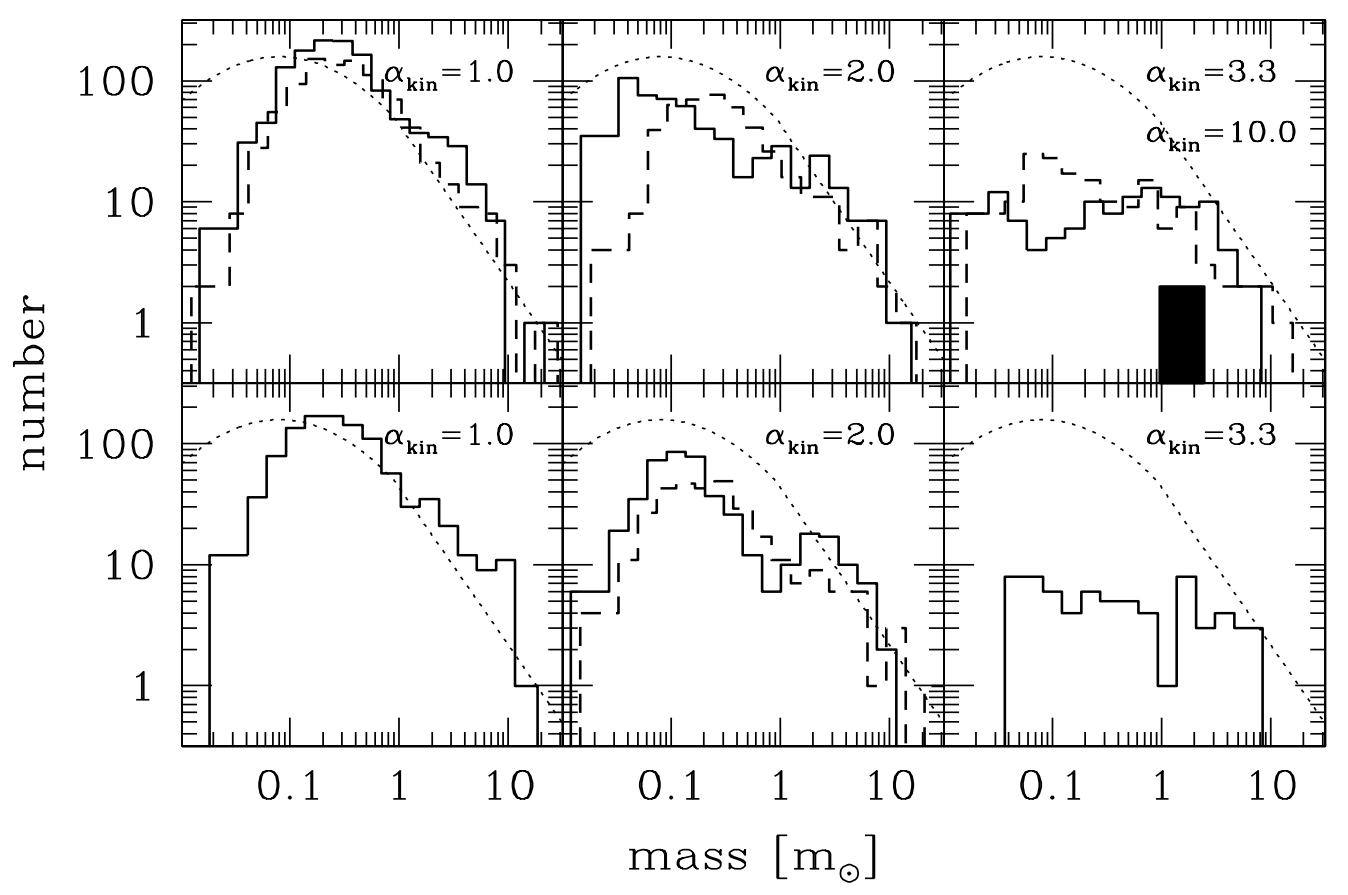

Figure 4. The mass functions of the sink particles for the simulations with $\alpha_{\text {kin }}=E_{\text {kin }} /\left|E_{\text {grav }}\right| \geqslant 1$, given after a time $t=2 t_{\text {ff }}$. The simulations with an isothermal EOS are shown in the upper panels, while those with the barotropic EOS are shown in the bottom panels. The long-dashed distributions show the results from a different realisation of the initial turbulent seed. The isothermal $\alpha_{\text {kin }}=0.1$ distribution (filled region) is shown alongside the $\alpha_{\text {kin }}=0.3$ results. The dotted distribution in each panel is the functional form of the IMF for the local field stars given by Chabrier (2003). Note that the mass resolution in all cases is $0.05 \mathrm{M}_{\odot}$, and those sinks with masses below this value are still accreting their neighbours.

tostellar system, and do not allow material to escape once it has been accreted. In reality stars will have winds and outflows, which are expected to lower the SFE of the protostellar core. However for low-mass stars, this effect is likely to be small (Wuchterl \& Klessen 2001). The formation of highermass stars may be less efficient due to the increased radiation pressure and presence of ionising winds Yorke \& Sonnhalter 2002; Krumholz, McKee \& Klein 2005). Therefore, if the internal SFE of a sink particle is a decreasing function of increasing mass, then the final stellar IMF would indeed be shallower than those presented in this study. To recover the observed Salpeter slope, the sink particle mass function would then need to be somewhat steeper. The sinks used in this study also have accretion radii that are larger than typical binary separations (Duquennoy \& Mayor 1991). As such, they are more representative of multiple systems, for systems smaller than the accretion radius $r_{\text {acc }}$ (as we point out in section 2). One would therefore expect the mass function to steepen somewhat for higher resolution.

\section{SUMMARY}

This paper examines the properties of the star formation in a series of $1000 \mathrm{M}_{\odot}$ clouds, in which the initial level of turbulent support is altered, with $\alpha_{\text {kin }}=E_{\text {kin }} /\left|E_{\text {grav }}\right|$ varying from 0.1 to 10 . We perform these calculations for two different EOSs, first assuming isothermality and then assuming a barotropic EOS, the latter of which is similar to that suggested by Larson (2005). The starting density in the barotropic EOS clouds is over an order of magnitude lower than that in the isothermal clouds.

We find that a wide range of star formation efficiencies is possible. In the simulations for which $\alpha_{\text {kin }} \geqslant 1$, the star formation efficiencies range from up to 60 percent, to as low as around 0.3 percent, after a time of 2 initial free-fall times. Since these calculations do not contain any model for the feedback from young stars, these SFEs are upper limits. The isothermal and barotropic equations of state yield similar results. We suggest that the very low level of star formation in the Maddelena cloud may be a result of the highly unbound structure that has been observed in this object Williams et al. (1994). In contrast, the simulations with $\alpha_{\text {kin }}=0.1$ are able to reach a star formation efficiency of around 35 percent, in only 1 free-fall time.

The mass functions of the 'sink' particles formed in the simulations are also found to be related to each cloud's initial dynamical state. In the clouds which start with $\alpha_{\text {kin }} \geqslant 1$, the mass functions of the sinks becomes progressively flatter with increasing initial energy in the turbulence. For the clouds with $\alpha_{\text {kin }}=1$ to 0.1 , the sink particle mass function are broadly similar to the stellar IMF. However we caution the reader that the sink particles used in this study are larger 


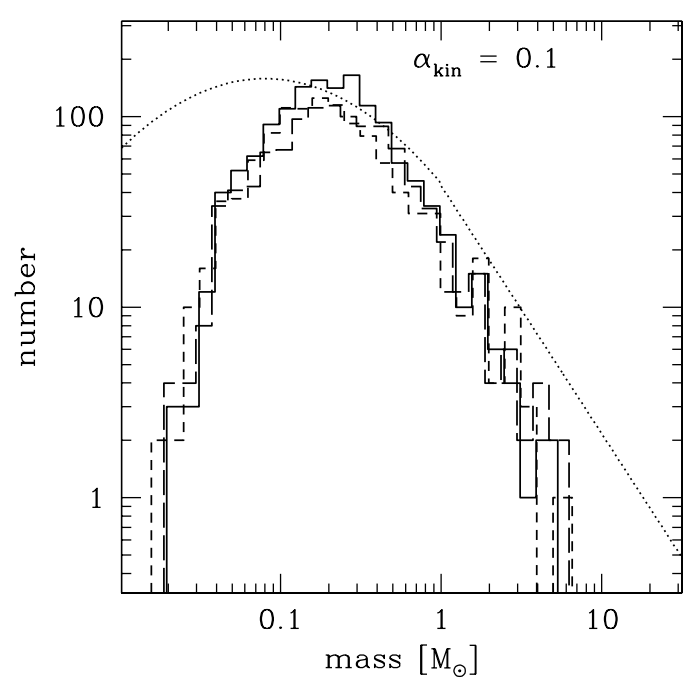

Figure 5. The sink mass functions for the weakly supported systems (those with $\alpha_{\text {kin }}=E_{\text {kin }} /\left|E_{\text {grav }}\right|=0.1$ ), given after a time $t=t_{\mathrm{ff}}$. The solid and dashed distributions show the mass function for the isothermal runs, for two different seeds, and the dotted distribution is for the simulation with the barotropic EOS.The dotted distribution in each panel is the functional form of the IMF for the local field stars given by Chabrier (2003). Note that the mass resolution in all cases is $0.05 \mathrm{M}_{\odot}$, and those sinks with masses below this value are still accreting their neighbours.

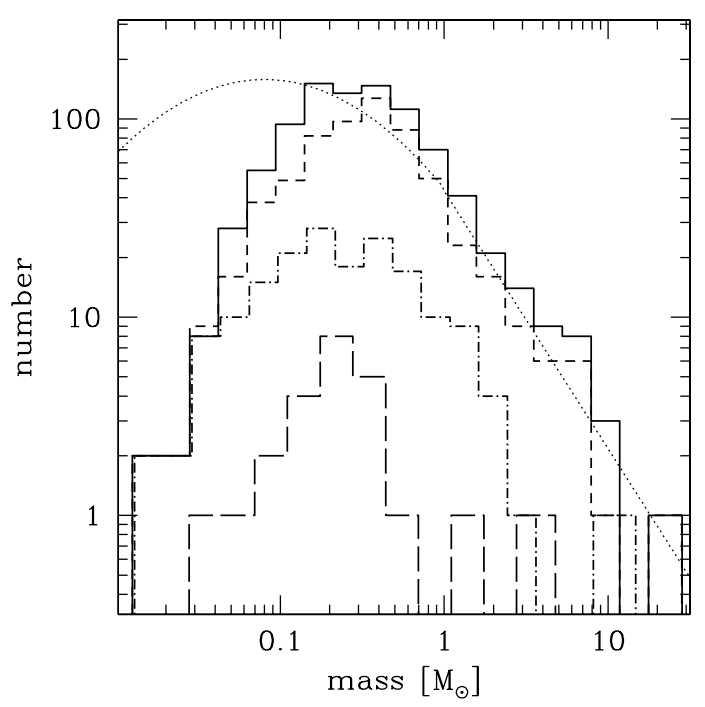

Figure 6. The mass function of sink particles, at four different instances, for isothermal simulation with velocity seed number 2 and $\alpha_{\text {kin }}=1$. The times for which the mass functions are shown are 0.75 (long-dashed), 1 (dot-dashed), 1.5 (short-dashed) and 2 (solid) initial free-fall times. Note that while the mass function at $2 t_{\mathrm{ff}}$ is the same as plotted in Figure 4 (top left panel, dashed distribution), the binning here is slightly larger, simply to make the plot easier to read. Again, the dotted line denotes the Chabrier (2003) IMF. than the typical binary system, and so are best interpreted as a system mass, which would be expected to be flatter than a true stellar mass function. All the features seen in the mass functions in this study are parallelled in both the EOSs that are employed.

We also point out that for $\alpha_{\text {kin }} \leqslant 1$, the general shape of the mass function is constant in time, including position of the turnover. This is an important feature of the mass function: regardless of when competitive accretion is interrupted by gas expulsion, the resulting mass function will be consistent with the observed distribution. As such, it does not suffer from the 'time-scale' problem, discussed in detail by Clark et al. (2007), which can arise when trying to map a pre-stellar core mass function to a star or system mass function.

We conclude that if competitive accretion is to play an important role in shaping the stellar IMF, then the majority of star formation needs to occur in bound, collapsing regions, at the scale of the cluster formation. However, the GMCs that host these cluster forming regions need not be globally bound entities. Within the GMC's unbound substructure, we predict that there can be a distributed mode of star formation, provided the gas in these structures has a sufficient number of Jeans masses. These stars are expected to exist in relative isolation, with a trend towards progressively more isolation in more unbound or dynamic regions. We predict that the IMF of this distributed population is flatter than that found in either the clustered environment or the local field star population.

\section{ACKNOWLEDGEMENTS}

We thank the anonymous referee for a close reading of the original manuscript which helped to clarify a number of issues. P.C.C. acknowledges support by the Deutsche Forschungsgemeinschaft (DFG) under grant KL 1358/5 and via the Sonderforschungsbereich (SFB) SFB 439, Galaxien im frühen Universum. We would like to thank Chris Rudge and Richard West at the UK Astrophysical Fluid Facility (UKAFF) for their tireless assistance and enthusiasm during the completion of this work.

\section{REFERENCES}

Allen L., Megeath S. T., Gutermuth R., Myers P. C., Wolk S., Adams F. C., Muzerolle J., Young E., Pipher J. L., 2007, in Reipurth B., Jewitt D., Keil K., eds, Protostars and Planets V The Structure and Evolution of Young Stellar Clusters. pp 361-376

André P., Belloche A., Peretto N., 2007, in prep

Ballesteros-Paredes J., 2006, MNRAS, 372, 443

Ballesteros-Paredes J., Hartmann L., Vázquez-Semadeni E., 1999, ApJ, 527, 285

Bally J., Stark A. A., Wilson R. W., Langer W. D., 1987, ApJL, 312, L45

Bate M. R., Bonnell I. A., Price N. M., 1995, MNRAS, 277, 362

Bate M. R., Burkert A., 1997, MNRAS, 288, 1060 
Benz W., 1990, in Numerical Modelling of Nonlinear Stellar Pulsations Problems and Prospects Smooth Particle Hydrodynamics - a Review. p. 269

Bhattal A. S., Francis N., Watkins S. J., Whitworth A. P., 1998, MNRAS, 297, 435

Bonnell I. A., Bate M. R., Clarke C. J., Pringle J. E., 2001a, MNRAS, 323, 785

Bonnell I. A., Bate M. R., Vine S. G., 2003, MNRAS, 343, 413

Bonnell I. A., Clarke C. J., Bate M. R., 2006, MNRAS, 368, 1296

Bonnell I. A., Clarke C. J., Bate M. R., Pringle J. E., 2001b, MNRAS, 324, 573

Bonnell I. A., Dobbs C. L., Robitaille T. P., Pringle J. E., 2006, MNRAS, 365, 37

Bonnell I. A., Vine S. G., Bate M. R., 2004, ArXiv Astrophysics e-prints

Carpenter J. M., 2000, AJ, 120, 3139

Chabrier G., 2003, PASP, , 115, 763

Chapman S., Pongracic H., Disney M., Nelson A., Turner J., Whitworth A., 1992, Nature, 359, 207

Clark P. C., Bonnell I. A., 2004, MNRAS, 347, L36

Clark P. C., Bonnell I. A., 2005, MNRAS, 361, 2

Clark P. C., Bonnell I. A., Zinnecker H., Bate M. R., 2005, MNRAS, 359, 809

Clark P. C., Klessen R. S., Bonnell I. A., 2007, MNRAS, 379,57

Clarke C. J., 1999, MNRAS, 307, 328

Dame T. M., Elmegreen B. G., Cohen R. S., Thaddeus P., 1986, ApJ, 305, 892

Dobbs C. L., Bonnell I. A., Pringle J. E., 2006, MNRAS, 371,1663

Doroshkevich A. G., 1980, Astronomicheskii Zhurnal, 57, 259

Duquennoy A., Mayor M., 1991, A\&A, 248, 485

Elmegreen B. G., 2000, ApJ, 530, 277

Elmegreen B. G., Elmegreen D. M., 1978, ApJ, 220, 1051

Elmegreen B. G., Scalo J., 2004, ARA\&A, 42, 211

Gittins D. M., Clarke C. J., Bate M. R., 2003, MNRAS, 340,841

Glover S. C. O., Mac Low M.-M., 2007a, ApJS, 169, 239

Glover S. C. O., Mac Low M.-M., 2007b, ApJ, 659, 1317

Hartmann L., Ballesteros-Paredes J., Bergin E. A., 2001, ApJ, 562, 852

Heitsch F., Mac Low M.-M., Klessen R. S., 2001, ApJ, 547, 280

Heyer M. H., Brunt C. M., 2004, ApJL, 615, L45

Jappsen A.-K., Klessen R. S., Larson R. B., Li Y., Mac Low M.-M., 2005, A\&A, 435, 611

Klessen R. S., 2001, ApJ, 556, 837

Klessen R. S., Ballesteros-Paredes J., Vázquez-Semadeni E., Durán-Rojas C., 2005, ApJ, 620, 786

Klessen R. S., Burkert A., 2000, ApJS, 128, 287

Klessen R. S., Burkert A., 2001, ApJ, 549, 386

Klessen R. S., Burkert A., Bate M. R., 1998, ApJL, 501, L205

Klessen R. S., Heitsch F., Mac Low M., 2000, ApJ, 535, 887

Kroupa P., 2002, Science, 295, 82

Kroupa P., Gilmore G., Tout C. A., 1991, MNRAS, 251, 293
Krumholz M. R., McKee C. F., Klein R. I., 2005, ApJL, 618, L33

Krumholz M. R., Tan J. C., 2007, ApJ, 654, 304

Lada C. J., Lada E. A., 2003, ARA\&A, 41, 57

Larson R. B., 1981, MNRAS, 194, 809

Larson R. B., 2005, MNRAS, 359, 211

Larson R. B., 2007, ArXiv Astrophysics e-prints

Lubow S. H., Pringle J. E., 1993, MNRAS, 263, 701

Mac Low M., Klessen R. S., 2004, Reviews of Modern Physics, 76, 125

Mac Low M., Klessen R. S., Burkert A., Smith M. D., 1998, Physical Review Letters, 80, 2754

Meyer M. R., Adams F. C., Hillenbrand L. A., Carpenter J. M., Larson R. B., 2000, Protostars and Planets IV, p. 121

Monaghan J. J., 1992, ARA\&A, 30, 543

Monaghan J. J., 2005, Reports of Progress in Physics, 68, 1703

Myers P. C., 1983, ApJ, 270, 105

Myers P. C., Gammie C. F., 1999, ApJL, 522, L141

Pringle J. E., Allen R. J., Lubow S. H., 2001, MNRAS, 327,663

Salpeter E. E., 1955, ApJ, 121, 161

Solomon P. M., Rivolo A. R., Barrett J., Yahil A., 1987, ApJ, 319, 730

Vázquez-Semadeni E., Ballesteros-Paredes J., Klessen R. S., 2003, ApJL, 585, L131

Vazquez-Semadeni E., Passot T., Pouquet A., 1995, ApJ, 441, 702

Vázquez-Semadeni E., Ryu D., Passot T., González R. F., Gazol A., 2006, ApJ, 643, 245

Williams J. P., de Geus E. J., Blitz L., 1994, ApJ, 428, 693

Wuchterl G., Klessen R. S., 2001, ApJL, 560, L185

Yorke H. W., Sonnhalter C., 2002, ApJ, 569, 846

Zinnecker H., 1982, New York Academy Sciences Annals, 395, 226

Zinnecker H., Yorke H. W., 2007, ARA\&A, 45, 481 